\title{
Une tumeur colique sous-muqueuse
}

\section{A sub mucosal colonic tumour}

\author{
V. Bon Djemah $\cdot$ X. Treton \\ (C) Springer-Verlag France 2011
}

\section{Situation clinique}

Une patiente de 34 ans suivie pour une rectocolite hémorragique pancolique évoluant depuis 12 ans consulte pour l'apparition de douleurs abdominales modérées, sans modification du transit. Ses antécédents étaient principalement marqués par une infertilité, non explorée. Depuis cinq ans, la rectocolite hémorragique est quiescente, sous un traitement par dérivés salicylés. Il n'existe pas d'anomalie biologique. La coloscopie met en évidence une lésion sigmoïdienne, évoquant une tumeur sous-muqueuse, volumineuse mais non obstructive (Fig. 1). La muqueuse colique en regard de la lésion est sensiblement normale et les biopsies montrent des lésions de colite chronique peu active, dans le rectosigmoïde, y compris en zone tumorale. Aucune dysplasie n'est retrouvée sur les biopsies coliques étagées multiples. Le scanner abdominopelvien montre la lésion sigmoïdienne suivante (Fig. 2).

\section{Question}

Quelle est votre attitude pratique?

\section{Réponse}

Il s'agit d'une lésion tumorale sous-muqueuse découverte chez une patiente ayant une rectocolite hémorragique peu active, sans lésion dysplasique muqueuse. L'indication d'une colectomie segmentaire à visée diagnostique et thérapeutique des douleurs abdominales n'a pas été retenue, car :

- le risque de survenue d'une colite postopératoire (en cas de colectomie partielle pour RCH) est élevé ;

- les douleurs abdominales étaient modérées et ;

\footnotetext{
V. Bon Djemah $\cdot$ X. Treton $(\triangle)$

Service de gastroentérologie, MICI et assistance nutritive, hôpital Beaujon, 100, boulevard du Général-Leclerc,

F-92118 Clichy cedex, France

e-mail : xavier.treton@bjn.aphp.fr
}

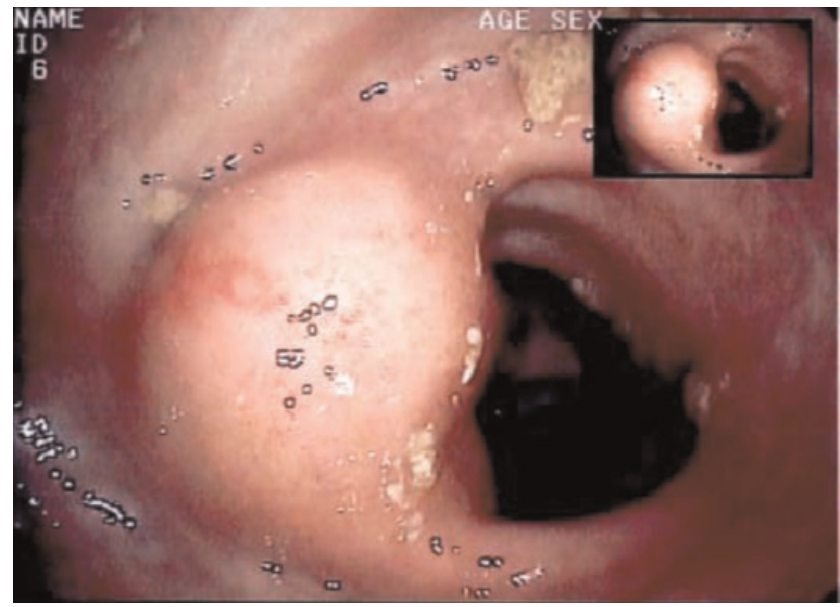

Fig. 1 Aspect endoscopique de la lésion sigmoïdienne

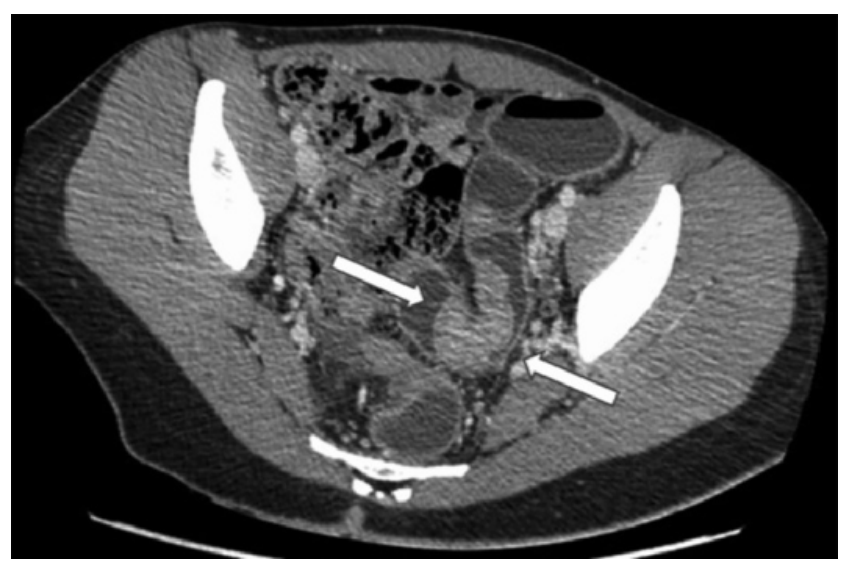

Fig. 2 Scannographie abdominale. Les flèches indiquent la lésion sigmoïdienne

- le diagnostic étiologique de la lésion non établi. La réalisation d'une échoendoscopie a permis de porter le diagnostic d'endométriose digestive profonde typique, et de proposer à la patiente un traitement et une surveillance médicale en première intention. 